\title{
Global formulation for interactive multiobjective optimization
}

\author{
Mariano Luque • Francisco Ruiz • \\ Kaisa Miettinen
}

Published online: 20 December 2008

(C) Springer-Verlag 2008

\begin{abstract}
Interactive methods are useful and realistic multiobjective optimization techniques and, thus, many such methods exist. However, they have two important drawbacks when using them in real applications. Firstly, the question of which method should be chosen is not trivial. Secondly, there are rather few practical implementations of the methods. We introduce a general formulation that can accommodate several interactive methods. This provides a comfortable implementation framework for a general interactive system. Besides, this implementation allows the decision maker to choose how to give preference information to the system, and enables changing it anytime during the solution process. This change-of-method option provides a very flexible framework for the decision maker.
\end{abstract}

Keywords Multiple criteria decision making - Multiple objectives · Interactive methods $\cdot$ Preference information

\section{Introduction}

During the years, many methods have been suggested for solving multiobjective optimization problems (see, e.g., Hwang and Masud 1979; Miettinen 1999; Sawaragi et al.

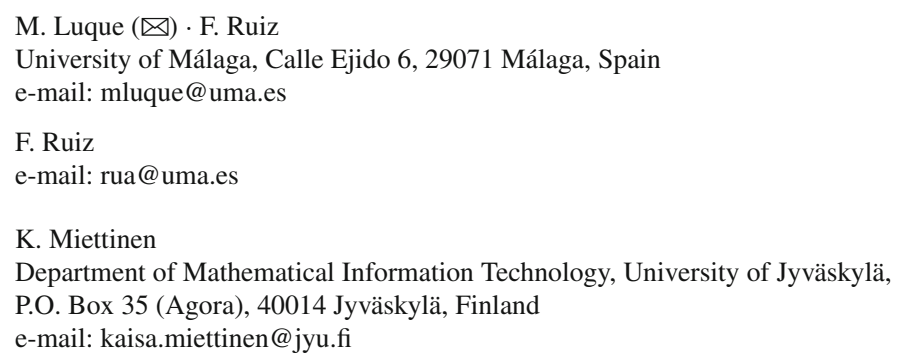


1985) where the aim is to find the most preferred solution in the presence of several conflicting objectives. The task of multiobjective optimization methods is to support a decision maker (DM) in formulating one's preferences and identifying the best of many Pareto optimal solutions. Different multiobjective optimization methods use a variety of forms of preference information and versatile techniques in generating Pareto optimal solutions. Because the task is to support a DM, so-called interactive approaches have turned out to be very useful and applicable. For this reason, many interactive methods, based on different solution philosophies, have been developed.

In interactive multiobjective optimization methods, a solution pattern is formed and repeated several times and at each iteration the DM can direct the search towards such Pareto optimal solutions that are interesting. The DM gets to see some information about the feasible Pareto optimal solutions available (and maybe some other information describing the trade-offs in the problem) and can specify preference information in order to find more preferable solutions. The benefit of using an interactive method is that the DM has a chance to learn about the interrelationships between the objectives and what kind of solutions are feasible for the problem. This helps in having more realistic expectations about the solutions. On the other hand, the DM can concentrate on such solutions that seem interesting and, thus, it typically is sufficient to produce a relatively small amount of Pareto optimal solutions, which means low computational costs.

Because there are many interactive methods available, it is not always easy to select the method to be used. As pointed out by Kaliszewski (2004), it would be good to create environments (with a common interface) where different methods were present and the DM could freely select the method (and the way of specifying preference information) as well as switch between methods. There have been some attempts of formulating this kind of an environment. For example, Gardiner and Steuer (1994a,b) suggested a unified algorithm which incorporates nine to thirteen different methods. However, the implementation of this unified algorithm is not a straightforward task. This conclusion is supported by Kaliszewski (2004) with the statement that even though the idea was appealing, it has remained too complex for DMs. Romero (2001) presented another attempt to prepare a general optimization structure where several variants of goal programming as well as some other methods can be covered. However, the usefulness and benefits of this approach have not been demonstrated or discussed from the implementation point of view. One more generalized formulation is given in Vassileva et al. (2005) incorporating thirteen different subproblems used in different methods but the formulation is rather complex. As far as implementations are concerned, PROMOIN (Caballero et al. 2002) is an example of an environment where several interactive methods are included but there is no general problem formulation and each method must be implemented separately. Furthermore, the synchronous NIMBUS method (Miettinen and Mäkelä 2006) and its implementations WWW-NIMBUS ${ }^{\circledR}$ (http://nimbus.it.jyu.fi/) and IND-NIMBUS ${ }^{\circledR}$ (Miettinen 2006) contain scalarizing functions of different methods but the way to specify preferences is the same for all of them.

In this paper, we introduce a general formulation that covers eight interactive multiobjective optimization methods representing three (or actually four) different method types. Namely, the reference point (Wierzbicki 1980), GUESS (Buchanan 1997), 
NIMBUS (Miettinen and Mäkelä 1995, 2006), STEM (Benayoun et al. 1971), STOM (Nakayama and Sawaragi 1984), Chebyshev (Steuer and Choo 1983), SPOT (Sakawa 1982) and PROJECT (Luque et al. 2008) methods are covered. The advantage of this formulation is its simple and compact structure which enables easy implementation. Furthermore, the framework presented allows the DM to conveniently change the style of expressing preference information, that is, changing the method used. However, the DM is not supposed to know different multiobjective optimization methods and their specificities but can concentrate on the actual problem to be solved and must only decide which kinds of preferences one could provide in order to direct the solution process to a desired direction so that the most preferred solution can be identified. Based on the preference type used, the general interactive solution scheme will choose the most appropriate method(s) for each case. The flexible possibility of changing the method means that the DM is not restricted to one way of specifying preferences. In different phases of the solution process the DM may wish to approach the problem in different ways and this is now possible. For example, at the early stages, in the so-called learning phase, the DM may wish to get a general overview of the solutions available and later on, once an interesting region of solutions has been identified, the DM may wish to fine-tune one's preferences in a smaller neighborhood. Our framework supports the DM in this and the DM has easy access to methods representing different solution philosophies.

It has often been wondered in the literature why relatively few real life applications of multiobjective optimization have been reported. One possible explanation is that computer implementations of the plethora of methods developed are not always easily accessible. Another explanation could be that they and their user interfaces are too complex for real DMs (as mentioned, e.g., in Kaliszewski 2004). The benefit of our framework is that it is easy to implement. Thus, we can say that one of our motivations in this paper is to bridge the gap between methods developed and their real-world applicability.

In practice, our interactive solution scheme is used so that at each iteration of the solution process, the DM can decide the type of preferences one wishes to specify: a reference point (or a classification of objective functions), selecting from a set of Pareto optimal solutions or marginal rates of substitution (MRSs). Our formulation contains several methods in each method type and problems corresponding to the type selected are formed (by setting appropriate variable values in the general problem formulation) and solved and the DM can decide whether to consider one such solution or more solutions based on the same preference information. Then, the DM can fine-tune one's preferences and get new solutions or continue by changing the type of preference information specified.

Let us point out that if the DM so desires, after having specified preferences in some form and obtained solution(s), it is possible to deduce what preferences of some other type could have produced the same solution, see Luque et al. (2007). This property can easily be included in our solution framework.

The rest of this paper is organized as follows. We discuss some concepts and notations in Sect. 2 and introduce our general problem framework, that is, GLIDE, the global formulation for interactive multiobjective optimization and the interactive solution scheme based on GLIDE in Sect. 3. Section 4 is devoted to an example 
demonstrating how our formulation can be applied and, finally, we conclude in Sect. 5 .

\section{Concepts and notations}

We consider multiobjective optimization problems of the form

$$
\begin{array}{ll}
\operatorname{minimize} & \left\{f_{1}(\mathbf{x}), f_{2}(\mathbf{x}), \ldots, f_{k}(\mathbf{x})\right\} \\
\text { subject to } & \mathbf{x} \in S
\end{array}
$$

involving $k$ ( $\geq 2$ ) conflicting objective functions $f_{i}: S \rightarrow \mathbb{R}$ that we want to minimize simultaneously. The decision variables $\mathbf{x}=\left(x_{1}, \ldots, x_{n}\right)^{T}$ belong to the nonempty compact feasible region $S \subset \mathbb{R}^{n}$. Objective vectors in objective space $\mathbb{R}^{k}$ consist of objective values $\mathbf{f}(\mathbf{x})=\left(f_{1}(\mathbf{x}), \ldots, f_{k}(\mathbf{x})\right)^{T}$ and the image of the feasible region is called the feasible objective region $Z=\mathbf{f}(S)$.

In multiobjective optimization, objective vectors are optimal if none of their components can be improved without deteriorating at least one of the others. More precisely, a decision vector $\mathbf{x}^{\prime} \in S$ is said to be efficient if there does not exist another $\mathbf{x} \in S$ such that $f_{i}(\mathbf{x}) \leq f_{i}\left(\mathbf{x}^{\prime}\right)$ for all $i=1, \ldots, k$ and $f_{j}(\mathbf{x})<f_{j}\left(\mathbf{x}^{\prime}\right)$ for at least one index $j$. On the other hand, a decision vector $\mathbf{x}^{\prime} \in S$ is said to be weakly efficient for problem (1) if there does not exist another $\mathbf{x} \in S$ such that $f_{i}(\mathbf{x})<f_{i}\left(\mathbf{x}^{\prime}\right)$ for all $i=1, \ldots, k$ and $\mathbf{x}^{\prime}$ is said to be properly efficient if unbounded trade-offs are not allowed. The corresponding objective vectors $\mathbf{f}\left(\mathbf{x}^{\prime}\right)$ are called (weakly/properly) nondominated objective vectors. Note that the set of properly nondominated solutions is a subset of nondominated solutions which is a subset of weakly nondominated solutions. In what follows, we denote the current nondominated solution by $\mathbf{f}^{h}$.

Let us assume that for problem (1) the set of nondominated objective vectors contains more than one vector. Because it is often useful to know the ranges of objective vectors in the nondominated set, we calculate the ideal objective vector $\mathbf{z}^{\star}=$ $\left(z_{1}^{\star}, \ldots, z_{k}^{\star}\right)^{T} \in \mathbb{R}^{k}$ by minimizing each objective function individually in the feasible region, that is, $z_{i}^{\star}=\min _{\mathbf{x} \in S} f_{i}(\mathbf{x})=\min _{\mathbf{x} \in E} f_{i}(\mathbf{x})$ for all $i=1, \ldots, k$, where $E$ is the set of efficient solutions. This gives lower bounds for the objectives. The upper bounds, that is, the nadir objective vector $\mathbf{z}^{\text {nad }}=\left(z_{1}^{\text {nad }}, \ldots, z_{k}^{\text {nad }}\right)^{T}$, can be defined as $z_{i}^{\text {nad }}=\max _{\mathbf{x} \in E} f_{i}(\mathbf{x})$ for all $i=1, \ldots, k$. In practice, the nadir objective vector is usually difficult to obtain. Its components can be approximated using a pay-off table but in general this kind of an estimate is not necessarily too good (see, e.g., Miettinen 1999, Section 2.4, and references therein). As an alternative, we can ask from the DM the worst possible objective values one could consider and use them as components of the nadir objective vector. In this way, the solution process is not disturbed by the possibly weak approximation obtained from the pay-off table.

Furthermore, sometimes (as proposed in Steuer 1986, 473-474) a utopian objective vector $\mathbf{z}^{\star \star}=\left(z_{1}^{\star \star}, \ldots, z_{k}^{\star \star}\right)^{T}$ is defined as a vector strictly better than the ideal objective vector. Then we set $z_{i}^{\star \star}=z_{i}^{\star}-\varepsilon(i=1, \ldots, k)$, where $\varepsilon>0$ is a small real number. This vector can be considered instead of an ideal objective vector in order to avoid the case where ideal and nadir values are equal or very close to each other. In 
what follows, we assume that the set of nondominated objective vectors is bounded and that we have global estimates of the ranges of nondominated solutions available.

All nondominated solutions can be regarded as equally desirable in the mathematical sense and we need a DM to identify the most preferred one among them. A DM is a person who can express preference information related to the conflicting objectives. In this paper, it will be assumed that the solution process is carried out using some interactive method (the general features of this class of methods have been described in Sect. 1). Among other issues, these methods differ from each other in the kind of information asked from the DM at each iteration.

In this paper, we consider different styles of specifying preference information: reference levels (that is, levels that are regarded as desirable for the DM for each objective function; or classification, that is, a division of the objectives into classes depending on whether the DM wishes to improve, impair or maintain the current value), just choosing one solution among several ones, or MRSs, that is, the amount of decrement in the value of one objective function that compensates an infinitesimal increment in the value of another one, while the values of all the other objectives remain unaltered. In this paper we introduce a global formulation which can be transformed, by changing some parameters, into interactive methods belonging to all these three classes.

\section{GLIDE: a global formulation for interactive multiobjective optimization}

We want to define a global formulation for interactive multiobjective optimization techniques. For this purpose, we define a general scalarized formulation called the GLobal Interactive Decision Environment (GLIDE), which has been designed so that it can generate different interactive techniques by changing the values of its parameters. Although eight different methods obtained from GLIDE are reported in this paper, the global formulation can accommodate other interactive procedures as well. For the multiobjective optimization problem (1), the GLIDE problem is defined as follows

$$
(\text { GLIDE })\left\{\begin{array}{lll}
\text { minimize } & \alpha+\rho \sum_{i=1}^{k} \omega_{i}^{h}\left(f_{i}(\mathbf{x})-q_{i}^{h}\right) & \\
\text { subject to } & \mu_{i}^{h}\left(f_{i}(\mathbf{x})-q_{i}^{h}\right) \leq \alpha & (i=1, \ldots, k) \\
& f_{i}(\mathbf{x}) \leq \varepsilon_{i}^{h}+s_{\varepsilon} \cdot \Delta \varepsilon_{i}^{h} & (i=1, \ldots, k) \\
& \mathbf{x} \in S,
\end{array}\right.
$$

where $\mathbf{x} \in \mathbb{R}^{n}$ is a vector of decision variables, and $\alpha \in \mathbb{R}$ is an auxiliary variable (not a parameter), introduced to make the problem differentiable. In addition, we have several parameters $\left(\alpha, \rho, \omega_{i}^{h}, q_{i}^{h}, \mu_{i}^{h}, \varepsilon_{i}^{h}, s_{\varepsilon}\right.$ and $\left.\Delta \varepsilon_{i}^{h}\right)$ which are to be set depending on the kind of information the DM is willing to provide, and consequently, on the corresponding interactive method used. That is, by changing the values of these parameters (as described in Tables 1, 2, 3, 4, 5, 6, 7, 8, 9), the GLIDE problem is transformed into the (intermediate) single objective problems employed by the eight different interactive methods covered by the general formulation. 
Table 1 REF: parameters for the reference point method (here $i=1, \ldots, k)$

Table 2 REF: parameters for the GUESS method (here $i=1, \ldots, k)$

\begin{tabular}{llll}
\hline Weights & $\omega_{i}^{h}=1$ & $\mu_{i}^{h}=\frac{1}{z_{i}^{\text {nad }}-z_{i}^{\star \star}}$ & $\rho>0$ \\
Reference levels & $q_{i}^{h}=\hat{q}_{i}^{h}$ & & \\
Objective bounds & $\varepsilon_{i}^{h}=z_{i}^{\text {nad }}$ & $\Delta \varepsilon_{i}^{h}=0$ & $s_{\varepsilon}=0$ \\
\hline
\end{tabular}

\begin{tabular}{llll}
\hline Weights & $\omega_{i}^{h}=0$ & $\mu_{i}^{h}=\frac{1}{z_{i}^{\text {nad }}-\hat{q}_{i}^{h}}$ & \\
Reference levels & $q_{i}^{h}=\hat{q}_{i}^{h}$ & & \\
Objective bounds & $\varepsilon_{i}^{h}=z_{i}^{\text {nad }}$ & $\Delta \varepsilon_{i}^{h}=0$ & $s_{\varepsilon}=0$ \\
\hline
\end{tabular}

Table 3 CLASS: parameters for the NIMBUS method

\begin{tabular}{llll}
\hline Weights & $\omega_{i}^{h}=\frac{1}{z_{i}^{\text {nad }}-z_{i}^{\star \star}}$ & $\mu_{i}^{h}=\frac{1}{z_{i}^{\text {nad }}-z_{i}^{\star \star}}$ for $i \in I_{h}^{\leq}$ & $\rho>0$ \\
& $(i=1, \ldots, k)$ & $\mu_{i}^{h}=1$ for $i \in I_{h}^{=} \cup I_{h}^{\geq}$ & \\
Reference levels & $q_{i}^{h}=\hat{q}_{i}^{h}$ for $i \in I_{h}^{\leq}$ & $q_{i}^{h}=z_{i}^{\text {nad }}+1 \quad$ for $i \in I_{h}^{=} \cup I_{h}^{\geq}$ & $s_{\varepsilon}=0$ \\
Objective & $\varepsilon_{i}^{h}=\hat{q}_{i}^{h}$ for $i \in I_{h}^{\geq}$ & $\Delta \varepsilon_{i}^{h}=0(i=1, \ldots, k)$ & \\
Bounds & $\varepsilon_{i}^{h}=f_{i}^{h}$ for $i \in I_{h}^{\leq} \cup I_{h}^{=}$ & & \\
\hline
\end{tabular}

Table 4 CLASS: parameters for the STEP method

\begin{tabular}{|c|c|c|c|}
\hline Weights & $\omega_{i}^{h}=0(i=1, \ldots, k)$ & $\begin{array}{l}\mu_{i}^{h}=\frac{z_{i}^{\text {nad }}-z_{i}^{\star \star}}{\max \left\{\left|z_{i}^{\text {nad }}\right|,\left|z_{i}^{\star \star}\right|\right\}} \quad \text { for } i \in I_{h}^{\leq} \\
\mu_{i}^{h}=1 \quad \text { for } i \in I_{h}^{=} \cup I_{h}^{\geq}\end{array}$ & $\rho=0$ \\
\hline Reference levels & $q_{i}^{h}=z_{i}^{\star \star} \quad$ for $i \in I_{h}^{\leq}$ & $q_{i}^{h}=z_{i}^{\text {nad }}$ for $i \in I_{h}^{=} \cup I_{h}^{\geq}$ & \\
\hline Objective & $\varepsilon_{i}^{h}=\hat{q}_{i}^{h} \quad$ for $i \in I_{h}^{\geq}$ & $\Delta \varepsilon_{i}^{h}=0(i=1, \ldots, k)$ & $s_{\varepsilon}=0$ \\
\hline Bounds & $\varepsilon_{i}^{h}=f_{i}^{h} \quad$ for $i \in I_{h}^{\leq} \cup I_{h}^{=}$ & & \\
\hline
\end{tabular}

Therefore, if the optimal solution obtained is denoted by $\mathbf{x}^{h+1}$ and the corresponding objective vector by $\mathbf{f}^{h+1}=\mathbf{f}\left(\mathbf{x}^{h+1}\right)$, the (weak, proper) efficiency is guaranteed just as in the corresponding methods. Anyway, the following theorem collects general results about the efficiency of the optimal solutions of problem (2), depending on the values of some parameters.

Theorem 1 Let $\mathbf{x}^{h+1}$ be an optimal solution of problem (2). Then, the following statements hold:

(i) If $\rho>0, \omega_{i}^{h}>0$ and $\mu_{i}^{h}>0(i=1, \ldots, k)$, then $\mathbf{x}^{h+1}$ is an efficient solution of problem (1).

Table 5 SAMPLE: parameters for the Chebyshev method (here $i=1, \ldots, k$ )

\begin{tabular}{llll}
\hline Weights & $\omega_{i}^{h}=1$ & $\mu_{i}^{h}$ (random) & $\rho>0$ \\
Reference levels & $q_{i}^{h}=z_{i}^{\star \star}$ & & \\
Objective bounds & $\varepsilon_{i}^{h}=z_{i}^{\text {nad }}$ & $\Delta \varepsilon_{i}^{h}=0$ & $s_{\varepsilon}=0$ \\
\hline
\end{tabular}


Table 6 MRS: parameters for the calculation of the optimal KKT multipliers, option 1 (here $i=1, \ldots, k)$

Table 7 MRS: parameters for the calculation of the optimal KKT multipliers, option 2 (here $i=1, \ldots, k)$

\begin{tabular}{|c|c|c|c|}
\hline Weights & $\begin{array}{l}\omega_{i}^{h}=0(i \neq r) \\
\omega_{r}^{h}=1\end{array}$ & $\mu_{i}^{h}=0$ & $\rho=1$ \\
\hline Reference levels & $q_{i}^{h}=0$ & & \\
\hline Objective & $\varepsilon_{i}^{h}=f_{i}^{h}(i \neq r)$ & $\Delta \varepsilon_{i}^{h}=0$ & $s_{\varepsilon}=0$ \\
\hline Bounds & $\varepsilon_{r}^{h}=z_{r}^{\text {nad }}$ & & \\
\hline
\end{tabular}

Weights

Reference levels

Objective bounds $\omega_{i}^{h}=0$

$q_{i}^{h}=z_{i}^{\star \star}$

$\mu_{i}^{h}=\frac{1}{f_{i}^{h}-z_{i}^{\star \star}} \quad \rho=0$

$\varepsilon_{i}^{h}=z_{i}^{\text {nad }} \quad \Delta \varepsilon_{i}^{h}=0$ $s_{\varepsilon}=0$

Table 8 MRS: parameters for the SPOT method (here $i=1, \ldots, k$ )

\begin{tabular}{|c|c|c|c|}
\hline Weights & $\begin{array}{l}\omega_{i}^{h}=0(i \neq r) \\
\omega_{r}^{h}=1\end{array}$ & $\mu_{i}^{h}=0$ & $\rho=1$ \\
\hline Reference levels & $q_{i}^{h}=0$ & & \\
\hline Objective & $\varepsilon_{i}^{h}=f_{i}^{h}(i \neq r)$ & $\Delta \varepsilon_{i}^{h}=\lambda_{r i}^{h}-m_{r i}^{h}(i \neq r)$ & $s_{\mathcal{E}}($ varied $)$ \\
\hline Bounds & $\varepsilon_{r}^{h}=z_{r}^{\mathrm{nad}}$ & $\Delta \varepsilon_{r}^{h}=0$ & \\
\hline
\end{tabular}

Table 9 MRS: parameters for the PROJECT method (here $i=1, \ldots, k)$

\begin{tabular}{llll}
\hline Weights & $\omega_{i}^{h}=0$ & $\mu_{i}^{h}=\frac{1}{\left|\hat{q}_{i}^{h}-f_{i}^{h}\right|}$ & $\rho=0$ \\
Reference levels & $q_{i}^{h}=\hat{q}_{i}^{h}$ & & \\
Objective bounds & $\varepsilon_{i}^{h}=z_{i}^{\text {nad }}$ & $\Delta \varepsilon_{i}^{h}=0$ & $s_{\varepsilon}=0$ \\
\hline
\end{tabular}

(ii) If $\rho=0$ or all $\omega_{i}^{h}=0$, and $\mu_{i}^{h}>0(i=1, \ldots, k)$, then $\mathbf{x}^{h+1}$ is a weakly efficient solution of problem (1). Moreover, if $\mathbf{x}^{h+1}$ is a unique solution, then it is efficient.

(iiii) If $\rho>0, \omega_{i}^{h}>0$ and $\mu_{i}^{h}=0(i=1, \ldots, k)$, then $\mathbf{x}^{h+1}$ is efficient. If for any $j$ we have $\omega_{j}^{h}=0$, then $\mathbf{x}^{h+1}$ is a weakly efficient solution of problem (1).

\section{Proof}

(i) Let us suppose that $\rho>0, \omega_{i}^{h}>0$ and $\mu_{i}^{h}>0$ for all $i$ and that $\mathbf{x}^{h+1}$ is not efficient. Then, there exists a feasible solution $\mathbf{x}^{*} \in S$ such that $f_{i}\left(\mathbf{x}^{*}\right)=f_{i}^{*} \leq$ $f_{i}^{h+1}(i=1, \ldots, k)$ with some $j$ such that $f_{j}^{*}<f_{j}^{h+1}$. Therefore, we have for all $i=1, \ldots, k$

$$
\begin{aligned}
& f_{i}^{*} \leq f_{i}^{h+1} \leq \varepsilon_{i}^{h}+s_{\varepsilon} \cdot \Delta \varepsilon_{i}^{h}, \\
& \mu_{i}^{h}\left(f_{i}^{*}-q_{i}^{h}\right) \leq \mu_{i}^{h}\left(f_{i}^{h+1}-q_{i}^{h}\right) \leq \alpha \text { and } \\
& \rho \sum_{i=1}^{k} \omega_{i}^{h}\left(f_{i}^{*}-q_{i}^{h}\right)<\rho \sum_{i=1}^{k} \omega_{i}^{h}\left(f_{i}^{h+1}-q_{i}^{h}\right),
\end{aligned}
$$


which implies that $\mathbf{x}^{*}$ is feasible for problem (2), and the corresponding value of the objective function is better than the objective value of $\mathbf{x}^{h+1}$, which contradicts the fact that $\mathbf{x}^{h+1}$ is an optimal solution of (2). Thus, $\mathbf{x}^{h+1}$ must be efficient.

(ii) If $\rho=0$ or all $\omega_{i}^{h}=0$, and $\mu_{i}^{h}>0(i=1, \ldots, k)$, then problem (2) is equivalent to solving a minmax problem with a restricted feasible region. If $\mathbf{x}^{h+1}$ is not weakly efficient, then there exists a feasible solution $\mathbf{x}^{*} \in S$, such that $f_{i}\left(\mathbf{x}^{*}\right)<f_{i}\left(\mathbf{x}^{h+1}\right)(i=1, \ldots, k)$.

Therefore, for each $i=1, \ldots, k$, we have:

$$
\begin{aligned}
& f_{i}^{*}<f_{i}^{h+1} \leq \varepsilon_{i}^{h}+s_{\varepsilon} \cdot \Delta \varepsilon_{i}^{h}, \\
& \mu_{i}^{h}\left(f_{i}^{*}-q_{i}^{h}\right)<\mu_{i}^{h}\left(f_{i}^{h+1}-q_{i}^{h}\right) \leq \alpha .
\end{aligned}
$$

Thus, $\mathbf{x}^{*}$ is feasible for problem (2), and the corresponding value of the objective function is strictly better than the objective value of $\mathbf{x}^{h+1}$ (as in case (i)). Because of this contradiction, $\mathbf{x}^{h+1}$ must be weakly efficient. Using the same reasoning it follows that if $\mathbf{x}^{h+1}$ is a unique optimal solution of (2), then it is efficient.

(iii) If $\rho>0, \omega_{i}^{h}>0$ and $\mu_{i}^{h}=0(i=1, \ldots, k)$, the first block of constraints of problem (2) is actually a non-negativity condition on $\alpha$. Therefore, at the optimal solution $\alpha$ must be zero, and thus, problem (2) is equivalent to solving

$$
\begin{array}{ll}
\operatorname{minimize} & \sum_{i=1}^{k} \omega_{i}^{h} f_{i}(\mathbf{x}) \\
\text { subject to } & f_{i}(\mathbf{x}) \leq \varepsilon_{i}^{h}+s_{\varepsilon} \cdot \Delta \varepsilon_{i}^{h}(i=1, \ldots, k) \\
& \mathbf{x} \in S,
\end{array}
$$

which is equivalent to a weighted sum problem with a restricted feasible region, whose additional constraints are the same as in the previous case. Therefore, any feasible solution with better or at least the same objective values as in $\mathbf{x}^{h+1}$ (with at least one strictly better value) would be feasible for (2), and it would have a better objective function value. Here we again have a contradiction and, thus, $\mathbf{x}^{h+1}$ must be efficient. Analogously, if for some $j$ we have $\omega_{j}^{h}=0$, then $\mathbf{x}^{h+1}$ is weakly efficient.

It is important to point out that all the efficient (or properly efficient) solutions of problem (1) can be obtained using this global scalarized formulation with adequate values for the parameters. For example, if we set $\mu_{i}^{h}>0$ and $\varepsilon_{i}^{h}+s_{\varepsilon} \cdot \Delta \varepsilon_{i}^{h} \geq z_{i}^{\text {nad }}$ $(i=1, \ldots, k)$, we get the achievement scalarizing function defined by Wierzbicki (1980).

Besides giving a general problem formulation, we also want to design a flexible global interactive solution scheme which allows the DM to choose the kind of information one wants to provide at each iteration. This will make the decision process easier for the DM, who does not have to follow the same preference elicitation pattern during the whole solution process. Rather than that, the scheme is adapted to the way the DM 


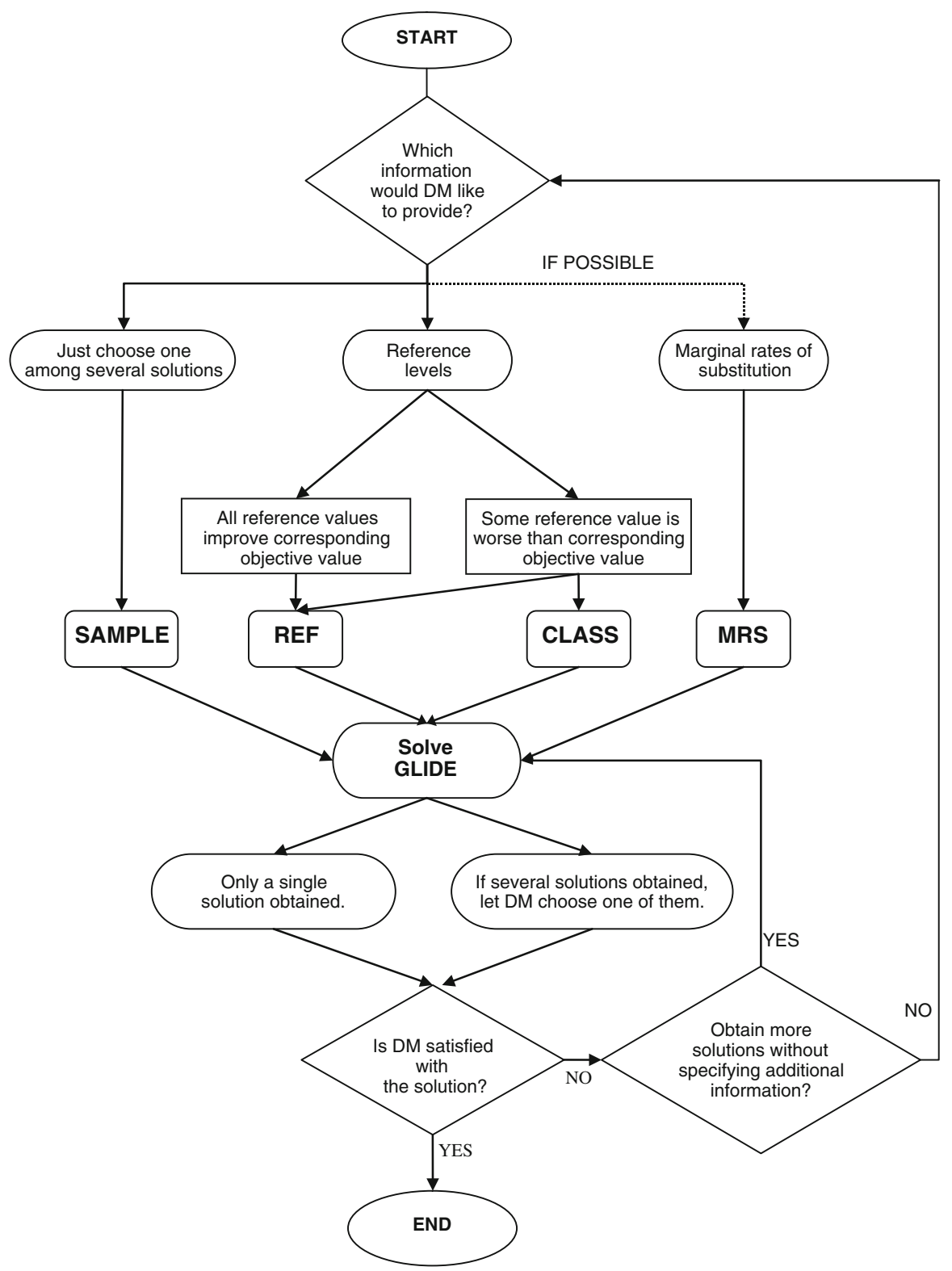

Fig. 1 Flowchart of the global interactive solution scheme for multiobjective optimization

prefers to provide preference information in, by changing the interactive method. To this end, based on the GLIDE formulation, we propose the global interactive solution scheme described in Fig. 1. Given the current solution, the DM can choose what kind of information (s)he wants to provide: just choose a solution among several ones, reference levels or marginal rates of substitution. The discontinuous line leading to 
the MRS option means that this case cannot be applied to all kinds of problems, as will be specified later in Sect. 3.3. Then the DM is asked to provide the corresponding preference information, and the parameters for GLIDE are set accordingly. Namely, if the reference point option is chosen, the reference point based methods (REF) or the classification methods (CLASS) can be used, as will be described in Sect. 3.1; if the DM wants to choose a solution among several ones, the corresponding methods (SAMPLE) are used as will be described in Sect. 3.2; and if the MRS option is chosen, then some methods of this type can be used, as will be described in Sect. 3.3.

Once the parameters have been set, problem (2) must be solved. If several solutions are generated, the DM has to choose one of them. The next step is to ask whether the DM is satisfied with this solution. If so, the solution process finishes. Otherwise, the DM can decide whether it is desirable to obtain more solutions without having to provide extra information. If the answer is positive, problem (2) is solved again with internally updated parameters values. To this end, several methods of the selected type are used. Finally, if the DM wishes to provide new kind of information, a new iteration is carried out.

In the implementation of this global formulation, it can be useful to allow the DM to save interesting solutions found during the solution process in a database (see, e.g., Jaszkiewicz and Slowiński 1999; Miettinen and Mäkelä 2006). In other words, the DM can save solutions that are potential candidates as a final solution although they do not seem quite satisfactory yet. In addition, given two already saved solutions, it may be interesting for the DM to generate intermediate solutions between them. This can be done by considering as reference points several points belonging to the segment which joins both the solutions, and adjusting the parameters as described in Sect. 3.1.

In what follows, we describe the different ways of specifying preference information that we consider and the corresponding interactive methods in more detail.

\subsection{Reference levels}

If the DM wishes to specify reference levels (also known as aspiration levels), given the current nondominated objective vector $\mathbf{f}^{h}$, the DM must provide such objective function values that should be reached. The point consisting of these reference levels is referred to as a reference point $\hat{\mathbf{q}}^{h}=\left(\hat{q}_{1}^{h}, \ldots, \hat{q}_{k}^{h}\right)$.

Because the current solution is nondominated, it is not possible to improve all objective function values simultaneously. However, the DM can specify a reference point without taking this specifically into account. Then, once a new solution is generated with a reference point based method, the DM can see which objective values did actually impair in order to let the others improve. However, if the DM wants to control the search more by specifying which objective functions are allowed to impair, we can utilize this information by using a classification based method. Thus, based on what kind of values the DM specifies, we differentiate two separate cases:

1. If each reference level improves the corresponding objective value, that is, $\hat{q}_{i}^{h} \leq$ $f_{i}^{h}(i=1, \ldots, k)$, then a reference point based method (REF) will be used. 
2. Otherwise, that is, if there exists $j$ such that $\hat{q}_{j}^{h}>f_{j}^{h}$, then a classification based method (CLASS) is chosen.

Note that based on the reasoning above, if classification based methods can be used, then also reference point based methods can be used but not vice versa (Miettinen and Mäkelä 2002).

\subsubsection{REF: generate first solution}

If the DM has specified reference levels such that a reference point based method is to be used, we use the reference point method (Wierzbicki 1980) to generate the first new nondominated solution to be shown to the DM. To this end, let us set the parameters for problem (2) as shown in Table $1 .{ }^{1}$ Parameter $\rho$ is the so-called augmentation coefficient. $^{2}$

Using these parameters, problem (2) is equivalent to solving the following problem:

$$
\begin{array}{ll}
\operatorname{minimize} & \alpha+\rho \sum_{i=1}^{k}\left(f_{i}(\mathbf{x})-\hat{q}_{i}^{h}\right) \\
\text { subject to } & \frac{f_{i}(\mathbf{x})-\hat{q}_{i}^{h}}{z_{i}^{\text {nad }}-z_{i}^{\star \star}} \leq \alpha \quad(i=1, \ldots, k) \\
& \mathbf{x} \in S .
\end{array}
$$

It must be pointed out that the weights $\mu_{i}^{h}$ in Table 1 have a pure normalizing role. It is also possible for the DM to use these coefficients in order to express preferences regarding the achievement of the reference levels. This possibility, which can accelerate in many cases the convergence of the method, is described in full detail in Luque et al. (2009), and from the implementation point of view, it only involves assigning different values to the weights $\mu_{i}^{h}$ in Table 1 .

\subsubsection{REF: generate more solutions}

If the DM wants to generate more solutions with the same preference information (that is, the same reference levels), we can consider a perturbation of the reference point,

\footnotetext{
${ }^{1}$ Let us note that the nadir value will be used in some parameters of this and other tables. If the nadir value appears in the parameter denoted by $\varepsilon$ in the general formulation, the aim is just to eliminate the corresponding constraint. Therefore, in this case, any big enough number can be used to approximate the nadir value. If, on the other hand, the nadir value appears in a weighting parameter (denoted by $\mu$ or $\omega$ ), then the final solution does depend on the approximated nadir value used. Nevertheless, the properties of the solution (regarding efficiency) hold anyway, and if the same approximation of the nadir value is used throughout the whole procedure, the solutions will be equally significant.

2 These augmented achievement scalarizing functions produce properly efficient solutions (see, e.g., Miettinen 1999, Sect. 3.4 and 3.5, and Wierzbicki 1986, for more details). It has also been shown that the augmentation terms may improve computational efficiency (Miettinen et al. 2006).
} 
as suggested by Wierzbicki (1980), for each $j \in\{1, \ldots, k\}$ as follows

$$
\begin{aligned}
& \Delta q_{j}^{h, j}=\left\|\hat{\mathbf{q}}^{h}-\mathbf{f}^{h, 0}\right\|_{2}, \\
& \Delta q_{i}^{h, j}=0(i=1, \ldots, k, \quad i \neq j),
\end{aligned}
$$

where $\mathbf{f}^{h, 0}$ is the objective vector corresponding to the solution generated by (3). In this way, up to $k$ new solutions can be generated by solving problem (2) with $\mathbf{q}^{h}=\hat{\mathbf{q}}^{h}+\Delta \mathbf{q}^{h, j}(j=1, \ldots, k)$ and keeping the other parameters as in Table 1.

If the DM still wants to see one more solution, the GUESS method (Buchanan 1997) can be used by setting the parameters for problem (2) as shown in Table 2.

In this case, problem (2) is equivalent to solving the problem

$$
\begin{array}{ll}
\operatorname{minimize} & \alpha \\
\text { subject to } & \frac{f_{i}(\mathbf{x})-\hat{q}_{i}^{h}}{z_{i}^{\text {nad }}-\hat{q}_{i}^{h}} \leq \alpha \quad(i=1, \ldots, k) \\
& \mathbf{x} \in S .
\end{array}
$$

Note that there is no augmentation term in the original method but in our formulation we can easily generate properly efficient solutions (instead of weakly efficient ones) by setting $\rho>0$ and $\omega_{i}^{h}=\mu_{i}^{h}(i=1, \ldots, k)$.

\subsubsection{CLASS: generate first solution}

If the DM specifies a reference point such that a classification based method can be used, we use the NIMBUS method (Miettinen and Mäkelä 1995, 2006) to generate the first solution. Let us define three classes of indices: $I_{h}^{\leq}=\left\{i \mid \hat{q}_{i}^{h}<f_{i}^{h}\right\}$, $I_{h}^{=}=\left\{i \mid \hat{q}_{i}^{h}=f_{i}^{h}\right\}$, and $I_{h}^{\geq}=\left\{i \mid \hat{q}_{i}^{h}>f_{i}^{h}\right\}$, and let us set the parameters for problem (2) as shown in Table 3.

Given the parameter values of Table 3, problem (2) can be expressed as

$$
\begin{array}{lll}
\text { minimize } & \alpha+\rho \sum_{i=1}^{k} \frac{f_{i}(\mathbf{x})}{z_{i}^{\text {nad }}-z_{i}^{\star \star}} & \\
\text { subject to } & \frac{f_{i}(\mathbf{x})-\hat{q}_{i}^{h}}{z_{i}^{\text {nad }}-z_{i}^{\star \star}} \leq \alpha & \left(i \in I_{h}^{\leq}\right) \\
& f_{i}(\mathbf{x}) \leq f_{i}^{h} & \left(i \in I_{h}^{\leq} \cup I_{h}^{=}\right) \\
& f_{i}(\mathbf{x}) \leq \hat{q}_{i}^{h} & \left(i \in I_{h}^{\geq}\right) \\
& \mathbf{x} \in S . &
\end{array}
$$

However, it may be necessary to clarify the reasoning behind the equivalence with problem (2) by showing an intermediate phase. Given that 


$$
\begin{aligned}
& \mu_{i}^{h}\left(f_{i}(\mathbf{x})-q_{i}^{h}\right)=1 \cdot\left(f_{i}(\mathbf{x})-\left(z_{i}^{\mathrm{nad}}+1\right)\right) \leq-1 \quad \text { for } i \in I_{h}^{=} \cup I_{h}^{\geq} \text {and } \\
& \mu_{j}^{h}\left(f_{j}(\mathbf{x})-q_{j}^{h}\right)=\frac{f_{j}(\mathbf{x})-\hat{q}_{j}^{h}}{z_{j}^{\text {nad }}-z_{j}^{\star \star}} \geq-1 \quad \text { for } j \in I_{h}^{\leq},
\end{aligned}
$$

then it follows that:

for any $i \in I_{h}^{=} \cup I_{h}^{\geq}$and $j \in I_{h}^{\leq}$we have $\mu_{i}^{h}\left(f_{i}(\mathbf{x})-\hat{q}_{i}^{h}\right) \leq \mu_{j}^{h}\left(f_{j}(\mathbf{x})-\hat{q}_{j}^{h}\right) \leq \alpha$

and, therefore, the optimal value for $\alpha$ is achieved at some constraint belonging to $I_{h}^{\leq}$. Therefore, the constraints regarding $I_{h}^{=} \cup I_{h}^{\geq}$can be eliminated from problem (2), and thus, we have the equivalent problem (4).

Note that in the original NIMBUS method, there are two more classes: $I_{h}^{<}$and $I_{h}^{\diamond}$ for functions to be improved as much as possible and functions that are allowed to change freely for a while, respectively. Anyway, if a reference level $\hat{q}_{i}^{h}$ is set equal to the corresponding ideal objective value $z_{i}^{\star}$, then this index could be considered as belonging to $I_{h}^{<}$. In the same way, if any reference level $\hat{q}_{j}^{h}$ is set equal to the corresponding nadir objective value $z_{i}^{\text {nad }}$, then this index could be considered as belonging to $I_{h}^{\diamond}$. For further details, see Miettinen (1999, Section 5.12), and Miettinen and Mäkelä (1995, 1999, 2000, 2006).

\subsubsection{CLASS: generate more solutions}

The GLIDE formulation can accommodate two other classification based methods: STEM and STOM. In order to obtain the STEP method (STEM) (Benayoun et al. 1971), let us define classes $I_{h}^{\leq}, I_{h}^{=}$, and $I_{h}^{\geq}$as in the NIMBUS method, and let us set the parameters for problem (2) as shown in Table 4.

Using a similar reasoning as in the previous method, problem (2) takes the following equivalent form:

$$
\begin{aligned}
& \text { minimize } \alpha \\
& \text { subject to } \frac{z_{i}^{\text {nad }}-z_{i}^{\star \star}}{\max \left\{\left|z_{i}^{\text {nad }}\right|,\left|z_{i}^{\star \star}\right|\right\}}\left(f_{i}(\mathbf{x})-z_{i}^{\star \star}\right) \leq \alpha \quad\left(i \in I_{h}^{\leq}\right) \\
& f_{i}(\mathbf{x}) \leq f_{i}^{h} \quad\left(i \in I_{h}^{\leq} \cup I_{h}^{=}\right) \\
& f_{i}(\mathbf{x}) \leq \hat{q}_{i}^{h} \quad\left(i \in I_{h}^{\geq}\right) \\
& \mathbf{x} \in S \text {, }
\end{aligned}
$$

which corresponds to STEM modified for nonlinear problems (Eschenauer et al. 1990). (Note that the original method was for linear problems only.)

Finally, the satisficing trade-offmethod (STOM) by Nakayama and Sawaragi (1984) can also be obtained from the GLIDE formulation by setting $q_{i}^{h}=z_{i}^{\star \star}$ and $\mu_{i}^{h}=$ $\frac{1}{\hat{q}_{i}^{h}-z_{i}^{\star \star}}(i=1, \ldots, k)$. All the other parameter values are the same as in the GUESS method in Table 2. (Under certain assumptions, the original STOM offers a 
possibility to calculate reference levels for objectives in $I_{h}^{\geq}$using sensitivity analysis, see Nakayama and Sawaragi (1984). However, we do not utilize it here because in our formulation we assume that the DM specifies all reference levels.)

Let us point out that if the DM wants to see more nondominated solutions after the solutions of NIMBUS, STEM and STOM have been generated, we can use the reference point based methods described earlier.

\subsection{SAMPLE: several solutions}

\subsubsection{SAMPLE: generate first solution}

If the DM wants only to compare a set of nondominated solutions, we use the Chebyshev method by Steuer and Choo (1983) (also known as the interactive weighted Tchebycheff method). To this end, the DM is asked to decide the number of solutions, $N S$, to be shown. Then, a sample of weight vectors (with all coordinates ranging between 0 and 1) will be randomly generated. For each vector of weights $\boldsymbol{\mu}^{h}$, let us set the parameters for problem (2) as shown in Table 5.

This problem is equivalent to solving:

$$
\begin{array}{ll}
\text { minimize } & \alpha+\rho \sum_{i=1}^{k}\left(f_{i}(\mathbf{x})-z_{i}^{\star \star}\right) \\
\text { subject to } & \mu_{i}^{h}\left(f_{i}(\mathbf{x})-z_{i}^{\star \star}\right) \leq \alpha \quad(i=1, \ldots, k) \\
& \mathbf{x} \in S,
\end{array}
$$

and a corresponding objective vector is obtained. (Actually, more than NS solutions can be generated and then filtered in order to obtain the NS most different objective vectors to be shown to the DM; see Steuer and Choo (1983) for further details about the random generation of weights and the filtering process).

If the DM wants to generate more concentrated solutions (for example solutions focused on an area around a given previously saved solution), the Chebyshev method offers the possibility to reduce the weights space using a reduction factor. Again, see Steuer and Choo (1983) for further details on this option. Finally, if the DM wants to generate another set of solutions, a new random generation process can be carried out using the same method (and therefore, the same parameters but new random weights). ${ }^{3}$

\subsection{MRS: marginal rates of substitution}

If the DM desires to specify marginal rates of substitution, then, given the current objective vector $\mathbf{f}^{h}$, the DM must choose a reference objective function $f_{r}$, and then provide MRSs $m_{r i}^{h}$ comparing each objective function to $f_{r}(i=1, \ldots, k, i \neq r)$. This information can be approximated in the following way. Starting from $\mathbf{f}^{h}$, the DM

\footnotetext{
3 If the random process is well designed and there are enough different solutions, the new solutions obtained are expected to be different from the previous ones.
} 
is required to provide the amount $\Delta f_{i}^{h}$ to be improved on the value of objective function $f_{i}$ that can exactly offset the given amount $\Delta f_{r}^{h}$ to be worsened of the reference objective $f_{r}$. Then, these amounts allow us to approximate the MRSs as

$$
m_{r i}^{h}=\frac{\Delta f_{r}^{h}}{\Delta f_{i}^{h}} \quad(i=1, \ldots, k) .
$$

As previously mentioned, MRS methods cannot be applied to any kind of problem. In what follows, we will state the conditions under which the different methods are assured to work properly. It is strongly recommended not to use this kind of methods unless one can somehow be sure that the corresponding conditions are fulfilled. To begin with, optimal Karush-Kuhn-Tucker (KKT) multipliers have to be calculated at the current solution. To this end, some regularity condition (constraint qualification) has to be satisfied at every iteration.

The optimal KKT multipliers can be calculated in two different ways. In option 1, it is also necessary that second order optimality conditions (Chankong and Haimes 1983, Chapter 4) are satisfied at the current solution. If this holds, the following problem is solved:

$$
\begin{array}{ll}
\operatorname{minimize} & f_{r}(\mathbf{x}) \\
\text { subject to } & f_{i}(\mathbf{x}) \leq f_{i}^{h} \quad(i=1, \ldots, k, i \neq r) \\
& \mathbf{x} \in S,
\end{array}
$$

which is obtained from problem (2) by setting the parameters as shown in Table 6.

Let us denote by $\lambda_{r i}^{h}$ the optimal KKT multipliers corresponding to the constraints set on objective functions (i.e., $f_{i}(\mathbf{x}) \leq f_{i}^{h}, i \neq r$ ). Furthermore, we set $\lambda_{r r}^{h}:=1$.

Option 2 to obtain the optimal KKT multipliers, which does not assume second order optimality conditions (Yang 1999), is by solving the following minmax problem

$$
\begin{array}{ll}
\operatorname{minimize} & \alpha \\
\text { subject to } & \frac{1}{f_{i}^{h}-z_{i}^{\star \star}}\left(f_{i}(\mathbf{x})-z_{i}^{\star \star}\right) \leq \alpha \quad(i=1, \ldots, k)
\end{array}
$$

which can also be obtained from problem (2) by considering the parameter values given in Table 7. Again, we denote by $\lambda_{r i}^{h}$ the optimal KKT multipliers corresponding to the constraints $\left(1 /\left(f_{i}^{h}-z_{i}^{\star \star}\right)\right)\left(f_{i}(\mathbf{x})-z_{i}^{\star \star}\right) \leq \alpha(i=1, \ldots, k)$.

\subsubsection{MRS: generate first solution}

The first solution based on MRSs is an adaptation of the SPOT method (Sakawa 1982). This adaptation does not make use of the proxy function (whose estimation goes beyond the reach of the GLIDE formulation). This method is applicable if the objective functions are twice continuously differentiable and convex, and the feasible set is convex. Besides, the existence of a continuously differentiable implicit value 
function of the DM, which is strictly decreasing and concave, is also assumed. Let us set the parameters for problem (2) as shown in Table 8.

Then, problem (2) is equivalent to solving the following problem:

$$
\begin{array}{ll}
\text { minimize } & f_{r}(\mathbf{x}) \\
\text { subject to } & f_{i}(\mathbf{x}) \leq f_{i}^{h}+s_{\varepsilon} \cdot\left(\lambda_{r i}^{h}-m_{r i}^{h}\right) \quad(i=1, \ldots, k, i \neq r) \\
& \mathbf{x} \in S .
\end{array}
$$

Several values for $s_{\varepsilon}$ are set and, in this way, different solutions are obtained by solving problem (7). The DM is then asked to choose one of them. The variation interval for $s_{\varepsilon}$ must be chosen in such a way that problem (7) is assured to have feasible solutions. To this end, it is necessary to calculate an upper bound for $s_{\varepsilon}$, denoted by $\bar{s}_{\varepsilon}$. Let $\bar{s}_{\varepsilon}$ be the smallest value of $s_{\varepsilon}$ such that some coordinate of $\varepsilon_{i}^{h}+s_{\varepsilon} \cdot \Delta \varepsilon_{i}^{h}$ reaches its corresponding ideal value. If problem (7), for $s_{\varepsilon}=\bar{s}_{\varepsilon}$, has feasible solutions, then $\bar{s}_{\varepsilon}$ is an appropriate upper bound. If not, we reduce $\bar{s}_{\varepsilon}$, multiplying it by a number between 0 and 1 (usually, this number is chosen between 0.5 and 1), and we check the feasibility again. The process continues until a valid value for $\bar{s}_{\varepsilon}$ is found. Then, several values between 0 and $\bar{s}_{\varepsilon}$ are selected and the corresponding problem (7) is solved.

\subsubsection{MRS: generate more solutions}

Finally, the algorithm used to generate alternative solutions under the MRS option is a modification of the GRIST method (Yang 1999), called PROJECT (Luque et al. 2008). In this method, the objective functions are assumed to be continuously differentiable, and the efficient set is assumed to be connected. Given the current objective vector and the MRSs between the objective functions, an approximation of the normalized value function gradient is calculated and a normal vector to the current objective vector is given by the optimal KKT multipliers mentioned above. The projection of this vector onto the tangent plane of the efficient set can provide an ascending direction in the DM's value function that better approximates the feasible region. Starting from $\mathbf{f}^{h}$ and in the direction of this projection vector, several points are generated and shown the DM. To generate these points, we calculate the last point whose coordinates are equal to or smaller than the corresponding ideal objective values (i.e., the first point with at least one coordinate being equal to the corresponding ideal objective value). Then we calculate several points between this point and the current objective vector and show them to the DM who is supposed to choose one. Let us denote it by $\hat{\mathbf{q}}^{h}$. This point chosen by the DM, which is infeasible in the majority of cases, is used to generate a solution which obeys the MRSs by solving the following problem:

$$
\begin{array}{ll}
\operatorname{minimize} & \alpha \\
\text { subject to } & \frac{f_{i}(\mathbf{x})-\hat{q}_{i}^{h}}{\left|\hat{q}_{i}^{h}-f_{i}^{h}\right|} \leq \alpha \quad(i=1, \ldots, k) \\
& \mathbf{x} \in S,
\end{array}
$$


which can be obtained from the GLIDE formulation by setting the parameters as shown in Table 9. For further details about the determination of the normal vector and other features of the algorithm, see Yang (1999), Yang and Li (2002).

\section{Example}

Let us illustrate with an example how our interactive solution scheme with GLIDE can be utilized. The problem considered has been described in Narula and Weistroffer (1989) and also considered in Miettinen and Mäkelä (1997) and briefly described in Miettinen et al. (2006). We have a pollution problem of a river involving four objective functions and two variables in the form

$$
\begin{array}{ll}
\text { maximize } & f_{1}(\mathbf{x})=4.07+2.27 x_{1} \\
\text { maximize } & f_{2}(\mathbf{x})=2.60+0.03 x_{1}+0.02 x_{2}+\frac{0.01}{1.39-x_{1}^{2}}+\frac{0.30}{1.39-x_{2}^{2}} \\
\text { maximize } & f_{3}(\mathbf{x})=8.21-\frac{0.71}{1.09-x_{1}^{2}} \\
\text { minimize } & f_{4}(\mathbf{x})=-0.96+\frac{0.96}{1.09-x_{2}^{2}} \\
\text { subject to } & 0.3 \leq x_{1}, x_{2} \leq 1.0 .
\end{array}
$$

In this problem, a fishery and a city of medium size are polluting water in the river and, thus, there is both industrial and municipal waste in the river. The city is situated downstream from the fishery. There exist two treatment plants, one in the fishery and another in the city. The waste is described in pounds of biochemical oxygen demanding material (BOD) and the two decision variables represent the proportional amounts of BOD removed from water in the treatment plants.

Here the first two objective functions describe the quality of water after the fishery and after the city, respectively. Cleaning the water in the fishery decreases the return on investments and, thus the third objective function measures the percent return on investment at the fishery. Finally, cleaning the water in the city means addition to the tax rate in the city and this is represented in the last objective function. Therefore, the first three objective functions are to be maximized, while the last one is to be minimized. The ideal and nadir values of the four objective functions are displayed in Table 10. It can be observed that $f_{2}$ (quality of the water after the city) has the smallest variation range.

Table 10 Ideal and nadir values

\begin{tabular}{llll}
\hline Objective & & Ideal & Nadir \\
\hline$f_{1}$ & Maximize & 6.34 & 4.75 \\
$f_{2}$ & Maximize & 3.44 & 2.85 \\
$f_{3}$ & Maximize & 7.50 & 0.32 \\
$f_{4}$ & Minimize & 0.00 & 9.71 \\
\hline
\end{tabular}


Iteration 1. When we start solving the problem, let us assume that the DM wishes to get an overall picture of the problem with four different nondominated solutions. Therefore, the option 'just choose one among several solutions' is used, and the following nondominated solutions are obtained by solving the GLIDE problem (2) with the parameters given in Table 5 (with four sets of random weights):

$$
\begin{aligned}
& (5.56,2.87,7.13,0.00),(6.00,2.88,6.27,0.00), \\
& (5.80,3.02,6.82,0.95),(6.21,3.15,4.75,2.00) .
\end{aligned}
$$

Among these solutions, the DM chooses the last one: $(6.21,3.15,4.75,2.00)$. In relative terms, here the value of $f_{1}$ (water quality after the fishery) is the closest one to its ideal value.

Iteration 2. Next, the DM wishes to give MRSs, ${ }^{4}$ taking $f_{1}$ as the reference objective. If this objective is relaxed by 0.1 units, the DM says that this is compensated by an improvement of 0.3 units in $f_{2}$, or an improvement of 0.1 units in $f_{3}$, or an improvement of 0.05 units in $f_{4}$. This implies that we can roughly speaking say that the DM regards $f_{2}$ as (locally) less important than objective 1, objective 3 as (locally) equally important than objective 1 , and $f_{4}$ as (locally) more important than objective one. Therefore, problem (2) is solved using the parameters shown in Table 8 for four step sizes, and the following solutions are shown to the DM:

$$
\begin{aligned}
& (6.21,3.15,4.75,2.00),(6.18,3.09,5.05,1.38) \\
& (6.15,3.03,5.35,0.87),(6.12,2.95,5.65,0.39),
\end{aligned}
$$

who chooses the second one: $(6.18,3.09,5.05,1.38)$.

As it can be seen, the environmental objectives $f_{1}$ and $f_{2}$ have experimented a light decrease, while the economical ones $\left(f_{3}\right.$ and $\left.f_{4}\right)$ have been improved.

Iteration 3. Let us now assume that the DM wishes to set very optimistic reference levels for every objective, as follows:

$$
\hat{q}_{1}^{h}=6.2, \quad \hat{q}_{2}^{h}=3.2, \quad \hat{q}_{3}^{h}=6.0, \quad \hat{q}_{4}^{h}=1.0 .
$$

Therefore, the reference point option is selected, and the parameters for problem (2) are set as shown in Table 1. As a consequence, the following solution is obtained: $(6.16,3.14,5.29,1.97)$.

In this iteration, the quality of the water after the fishery $\left(f_{1}\right)$ has slightly decreased and the addition to the tax rate $\left(f_{4}\right)$ has increased significantly. On the other hand, both the quality of the water after the city $\left(f_{2}\right)$ and the return on investments $\left(f_{3}\right)$ have increased.

Iteration 4. Now the DM wishes to improve the economical objectives at the price of relaxing a little bit in the environmental ones. Therefore, the DM sets the following reference levels:

\footnotetext{
4 Note that for illustrative purposes we here use this option even though the problem does not satisfy the assumptions specified in Sect. 3.3.1.
} 

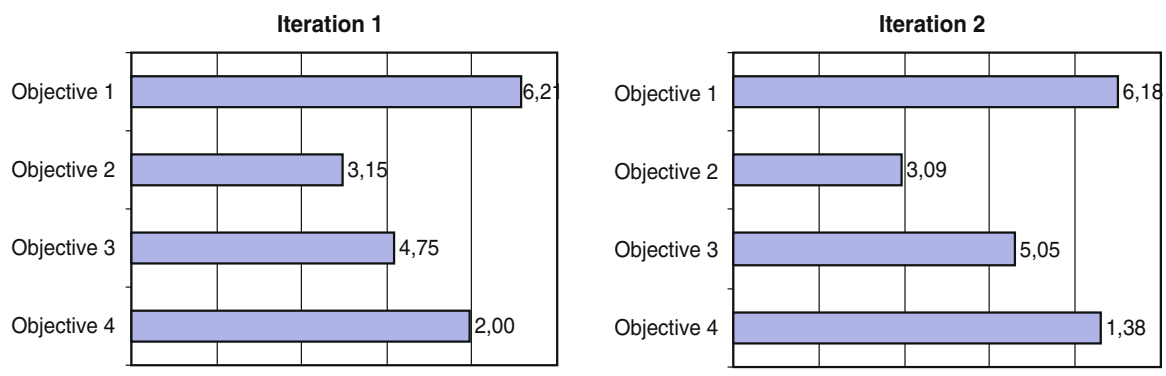

Iteration 3
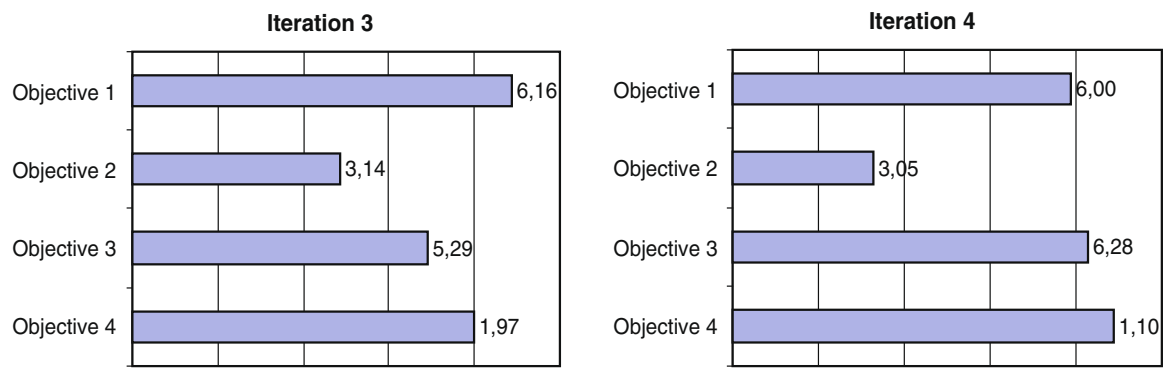

Fig. 2 Graphical display of the four iterations

$$
\hat{q}_{1}^{h}=6.0, \quad \hat{q}_{2}^{h}=3.0, \quad \hat{q}_{3}^{h}=6.0, \quad \hat{q}_{4}^{h}=1.5 .
$$

In this case, the classification based option is chosen and problem (2) is solved using the parameters shown in Table 3. The solution obtained is the following one: $(6.00,3.05,6.28,1.10)$.

As it can be seen, the quality of the water after the fishery is at its reference level and the quality of water after the city is slightly better than the reference level. On the other hand, the economical objectives have been improved beyond their reference levels. Let us assume that the DM decides to stop with this solution, which implies to remove the $85.02 \%$ of the BOD at the treatment plant of the fishery, and the $79.01 \%$ of the BOD at the treatment plant of the city. A graphical representation of the four iterations (with the solutions selected) can be seen in Fig. 2, where the bigger the horizontal bars are, the better the value of the corresponding objective is.

Finally, it must be pointed out that the purpose of this example is to illustrate the performance and flexibility of the global interactive solution scheme, and that is why four iterations have been carried out, all of them with different preference elicitation styles. Obviously, the DM does not have to change the type of preference information so often, but it is possible to ask for several solutions based on the information already specified and continue the solution process as long as desired.

\section{Conclusions}

In this paper, a global formulation which can accommodate several interactive multiobjective optimization methods has been introduced. The compact structure of this 
formulation takes the form of a general optimization problem with a set of parameters that have to be changed in order to obtain the different interactive methods supported. To be more specific, in this paper we show how the Reference Point, GUESS, NIMBUS, STEM, STOM, Chebyshev, SPOT and PROJECT methods can be derived from this general formulation (besides, other interactive methods like Klamroth and Miettinen 2008 could also be considered by choosing the corresponding parameters).

It is clear that one of the main features that an interactive decision aid tool must have in order to successfully tackle real problems is flexibility. This flexibility is understood as the ability of the system to adapt the solution process to the DM's wishes allowing one to choose the most appropriate way of providing information any time during the process. To this end, it is necessary to implement different interactive techniques which utilize different kinds of preference information. On the other hand, one of the weak points of the interactive multiobjective optimization field is the very limited number of practical and easy-to-use implementation.

We would like to emphasize the compactness of the formulation proposed, which makes it different from the other general counterparts previously published. The GLIDE formulation has been designed with two main aims. First, providing a comfortable decision aid environment for the DM, and second, aiding potential programmers to implement the interactive system.

From the point of view of the user, a general algorithm has been built where the DM only has to decide what type of preference information (reference levels, choosing a solution among some efficient solutions or MRSs) to provide at each iteration. The algorithm chooses the corresponding method and produces the solution(s). Given that more than one interactive method of each class is supported by the global formulation, it is also possible to generate several different solutions with the same information if the DM so wishes.

From the point of view of the programmer, the global formulation is complemented with tables with the values of the parameters of GLIDE for each of the methods considered. This provides a simple implementation framework that makes it easier to create an interactive system based on the GLIDE formulation.

Finally, a simple hypothetical illustrative example has been used to show the performance of the global formulation and of the interactive solution scheme proposed. Although a preliminary implementation has been developed in order to test the algorithm and to solve the example, our future research trends include a more versatile implementation of an interactive system based on GLIDE with a flexible and graphical user interface, and its application to real life problems.

Acknowledgments This research was partly supported by the Andalusian Regional Ministry of Innovation, Science and Enterprises (PAI group SEJ-445), the Spanish Ministry of Education and Science (MTM2006-01921) as well as Tekes, the Finnish Funding Agency for Technology and Innovation (the Technology Programme for Modelling and Simulation).

\section{References}

Benayoun R, de Montgolfier J, Tergny J, Laritchev O (1971) Programming with multiple objective functions: step method (STEM). Math Program 1(3):366-375 
Buchanan JT (1997) A naïve approach for solving MCDM problems: the GUESS method. J Oper Res Soc 48(2):202-206

Caballero R, Luque M, Molina J, Ruiz F (2002) PROMOIN: an interactive system for multiobjective programming. Int J Inform Technol Decis Making 1:635-656

Chankong V, Haimes YY (1983) Multiobjective decision making: theory and methodology. North-Holland, New York

Eschenauer HA, Osyczka A, Schäfer E (1990) Interactive multicriteria optimization in design process. In: Eschenauer H, Koski J, Osyczka A (eds) Multicriteria design optimization procedures and applications. Springer, Berlin, pp 71-114

Gardiner L, Steuer RE (1994a) Unified interactive multiple objective programming. Eur J Oper Res 74(3):391-406

Gardiner L, Steuer RE (1994b) Unified interactive multiple objective programming: an open architecture for accommodating new procedures. J Oper Res Soc 45(12):1456-1466

Hwang CL, Masud ASM (1979) Multiple objective decision making-methods and applications: a stateof-the-art survey. Springer, Berlin

Jaszkiewicz A, Slowiński R (1999) The 'light beam search' approach—an overview of methodology and applications. Eur J Oper Res 113:300-314

Kaliszewski I (2004) Out of the mist-towards decision-maker-friendly multiple criteria decision making support. Eur J Oper Res 158:293-307

Klamroth K, Miettinen K (2008) Integrating approximation and interactive decision making in multicriteria optimization. Oper Res 56(1):222-234

Luque M, Caballero R, Molina J, Ruiz F (2007) Equivalent information for multiobjective interactive procedures. Manage Sci 53(1):125-134

Luque M, Yang JB, Wong BYH (2008) PROJECT method for multiobjective optimisation based on gradient projection and reference point. IEEE Trans Syst Man Cybern-Part A (to appear)

Luque M, Miettinen K, Eskelinen P, Ruiz F (2009) Incorporating preference information in interactive reference point methods for multiobjective optimization. Omega 37(2):450-462

Miettinen K (1999) Nonlinear multiobjective optimization. Kluwer, Boston

Miettinen K (2006) IND-NIMBUS for demanding interactive multiobjective optimization. In: Trzaskalik $\mathrm{T}$ (ed) Multiple Criteria Decision Making '05. The Karol Adamiecki University of Economics in Katowice, Katowice, pp 137-150

Miettinen K, Mäkelä MM (1995) Interactive bundle-based method for nondifferentiable multiobjective optimization: NIMBUS. Optimization 34(3):231-246

Miettinen K, Mäkelä MM (1997) Interactive method NIMBUS for nondifferentiable multiobjective optimization problems. In: Climaco J (ed) Multicriteria analysis. Springer, Berlin, pp 310-319

Miettinen K, Mäkelä MM (1999) Comparative evaluation of some interactive reference point-based methods for multi-objective optimisation. J Oper Res Soc 50(9):949-959

Miettinen K, Mäkelä MM (2000) Interactive multiobjective optimization system WWW-NIMBUS on the Internet. Comput Oper Res 27(7-8):709-723

Miettinen K, Mäkelä MM (2002) On scalarizing functions in multiobjective optimization. OR Spectr 24(2):193-213

Miettinen K, Mäkelä MM (2006) Synchronous approach in interactive multiobjective optimization. Eur J Oper Res 170(3):909-922

Miettinen K, Mäkelä MM, Kaario K (2006) Experiments with classification-based scalarizing functions in interactive multiobjective optimization. Eur J Oper Res 175(2):931-947

Nakayama H, Sawaragi Y (1984) Satisficing trade-off method for multiobjective programming. In: Grauer M, Wierzbicki AP (eds) Interactive decision analysis. Springer, Berlin, pp 113-122

Narula SC, Weistroffer HR (1989) A flexible method for nonlinear multicriteria decisionmaking problems. IEEE Trans Syst Man Cybern 19(4):883-887

Romero C (2001) Extended lexicographic goal programming: a unified approach. Omega 29:63-71

Sakawa M (1982) Interactive multiobjective decision making by the sequential proxy optimization technique: SPOT. Eur J Oper Res 9(4):386-396

Sawaragi Y, Nakayama H, Tanino T (1985) Theory of multiobjective optimization. Academic Press, Orlando

Steuer RE (1986) Multiple criteria optimization: theory, computation and application

Steuer RE, Choo EU (1983) An interactive weighted Tchebycheff procedure for multiple objective programming. Math Program 26:326-344 
Vassileva M, Miettinen K, Vassilev V (2005) Generalized scalarizing problem for multicriteria optimization. IIT Working Papers IIT/WP-205, Institute of Information Technologies, Bulgaria

Wierzbicki AP (1980) The use of reference objectives in multiobjective optimization. In: Fandel G, Gal T (eds) Multiple criteria decision making, theory and applications. Springer, Berlin, pp 468-486

Wierzbicki AP (1986) On the completeness and constructiveness of parametric characterizations to vector optimization problems. OR Spectr 8(2):73-87

Yang JB (1999) Gradient projection and local region search for multiobjective optimization. Eur J Oper Res 112:432-459

Yang JB, Li D (2002) Normal vector identification and interactive tradeoff analysis using minimax formulation in multiobjective optimisation. IEEE Trans Syst Man Cybern A Syst Humans 32(3):305-319 